Musées, Patrimoine et Culture scientifiques et techniques

\title{
Verviers, au fil de l'eau et de la laine : histoire, tourisme et politique
}

Noémie Drouguet

\section{(2) OpenEdition \\ Journals}

Édition électronique

URL : http://journals.openedition.org/ocim/352

DOI : 10.4000/ocim.352

ISSN : 2108-646X

Éditeur

OCIM

Édition imprimée

Date de publication : 1 septembre 2008

Pagination : 18-21

ISSN : 0994-1908

Référence électronique

Noémie Drouguet, «Verviers, au fil de l'eau et de la laine : histoire, tourisme et politique », La Lettre de I'OCIM [En ligne], 119 | 2008, mis en ligne le 21 janvier 2011, consulté le 01 mai 2019. URL : http:// journals.openedition.org/ocim/352; DOI : 10.4000/ocim.352 
Verviers qui offre aux visiteurs, locaux ou de passage, deux infrastructures culturelles et touristiques de ce type, ainsi qu'un parcours urbain. Le centre touristique de la Laine et de la Mode (CTLM) et la maison de l'Eau réunissent en effet les traits que l'on reconnaît habituellement aux centres d'interprétation, et ils sont tous deux assez caractéristiques de la façon dont on les envisage en Wallonie, du point de vue de leur fonction et de leur conception.

\section{Portrait d'une ville}

Dès le XVII e siècle et jusqu'à la crise des années 1930, Verviers compte parmi les plus grands centres lainiers du monde. Après la Première Guerre mondiale, cette petite ville de l'Est de la Belgique devient capitale mondiale de la laine, avec Bradford (Angleterre). À la faveur d'une industrialisation extrêmement rapide, la modeste bourgade, dont les racines historiques ne remontent guère au-delà du $\mathrm{XVI}^{\mathrm{e}}$ siècle, se couvre de fouleries, filatures et autres draperies et la ville connaît une expansion exceptionnelle, grâce à la « bienveillance » de quelques dynasties d'industriels. Durant l'entre deux guerres, les affaires s'essoufflent, puis les années 1950 voient le déclin définitif de la production textile à Verviers et amorcent le début d'une longue période de morosité économique. Depuis une quinzaine d'années, la ville de 55000 habitants tente de présenter un nouveau visage sur les plans urbanistique et culturel, oubliant les balafres de l'industrie et mettant au contraire en valeur le passé lainier sous ses aspects les plus glorieux et prospères.

Limage positive que Verviers veut projeter d'ellemême passe par la création d'un « centre de valorisation touristique de l'industrie de la laine » : le centre touristique de la Laine et de la Mode, inauguré en $1999^{(2)}$. La ville a inscrit ce projet dans le cadre d'« Objectif 2 Meuse-Vesdre », de façon a obtenir des subsides européens et régionaux. Ce centre prend place dans une ancienne manufacture de draps datant du début du XVIII siècle, flanquée d'un hôtel de maître réservé à la direction. L'exposition se présente comme un «parcours spectacle» au long duquel le visiteur est guidé par un système d'écouteurs. Fil de laine raconte son histoire, depuis la tonte d'un mouton en Australie jusqu'à la trame d'un tissu produit à Verviers. Le visiteur passe de salle en salle dans des décors reconstitués : le bureau d'un administrateur de filature, les usines avec les machines, l'oiselerie où les ouvriers se retrouvent, le salon du tailleur... Sur le plan muséographique, l'accent est mis sur l'immersion et l'émotion grâce aux décors de grande qualité. Quelques objets, documents et machines ponctuent la première moitié du parcours. Pour la seconde moitié, Fil de laine cède sa place à Fil du temps, qui évoque l'évolution de l'habillement et de la mode depuis l'Antiquité jusqu'à nos jours. Des extraits de bandes dessinées reproduits en grand format, flanquées de quelques éléments de mobilier, illustrent les tendances du costume à quelques époques. Des mannequins vêtus de reproductions de tenues arborées par les héros dans les cases de BD ainsi que quelques téléviseurs sur lesquels défilent des extraits de films "d'époque » (Ben-Hur, Les liaisons dangereuses...) agrémentent mollement la visite de cette partie, dont la muséographie est beaucoup plus faible. L'ensemble du projet a été porté par Jeannot Kupper, de la société EuroCulture Production, spécialisée dans les expositions-spectacles (3). Cette entreprise convenait parfaitement à la philosophie du projet, qui a cherché d'emblée à se différencier d'un «musée de la laine », jugé plus ennuyeux, et qui a pour ambition de présenter "le meilleur d'une ville qui a connu un passé prestigieux et dont les ressources patrimoniales et touristiques méritent le détour»(4).

On l'aura compris, le but n'est pas de proposer un portrait équilibré de la ville et de son industrie déchue. Si les conditions de travail et les revendications des travailleurs ne sont pas évacuées, les problèmes sociaux liés à l'industrie de la laine sont évoqués de façon anecdotique. Les intentions politiques et muséographiques sont aux antipodes d'un écomusée, par exemple. Nulle envie ici de provoquer une adhésion et un investissement des habitants et de mobiliser le lieu en vecteur de transformation sociale pour favoriser une prise de conscience afin que la population saisisse par la connaissance de son histoire les rênes de sa destinée.

En plus de ce parcours permanent, le CTLM propose des expositions temporaires, sur des sujets qui n'entretiennent pas nécessairement de lien avec l'industrie lainière mais qui ont la capacité de capter un large public scolaire et familial. Des thèmes très populaires comme Claude François, les Playmobil, les albums de Martine ou d'Alix, le carnaval de Rio, le chocolat... servent essentiellement à fidéliser des visiteurs, qui ne reviendraient sans doute pas uniquement pour le parcours permanent, qui n'a pratiquement pas évolué depuis l'ouverture du centre. 


\section{La maison de l'Eau}

Ouverte plus récemment au public, la maison de l'Eau se profile comme le complément idéal du CTLM. Ce second centre d'interprétation est situé dans le même quartier et occupe également un bâtiment hautement représentatif de l'histoire lainière : la maison de Bonvoisin, témoignage architectural intéressant d'un atelier accolé à la maison d'un artisan. Il y est à nouveau question du glorieux passé lainier de la ville, à travers la Vesdre. Cette rivière qui traverse la ville est l'une des clés du succès de l'industrie textile : ses eaux acides présentaient les qualités idéales pour laver la laine et sa force motrice fut également utilisée pour actionner les machines. La création de ce second volet de l'histoire lainière, quelque peu redondant, est notamment liée à l'implantation à Verviers des sièges des principaux acteurs publics dans le domaine : la Société wallonne des Eaux (SWDE) et la Société publique de Gestion de l'Eau (SPGE), avant d'accueillir un centre de formation aux métiers de l'eau et un centre de gestion de l'eau. Verviers est désormais « capitale wallonne de l'eau » et veut le faire savoir.

Sur le plan des intentions muséographiques, le programme est assez proche de celui du CTLM : mettre en valeur le passé glorieux et entrevoir un avenir radieux en ce qui concerne l'eau et la Vesdre, jouer de présentations et de dispositifs spectaculaires, ancrer le message dans l'histoire locale pour terminer sur des généralités un peu banales, telles qu’une

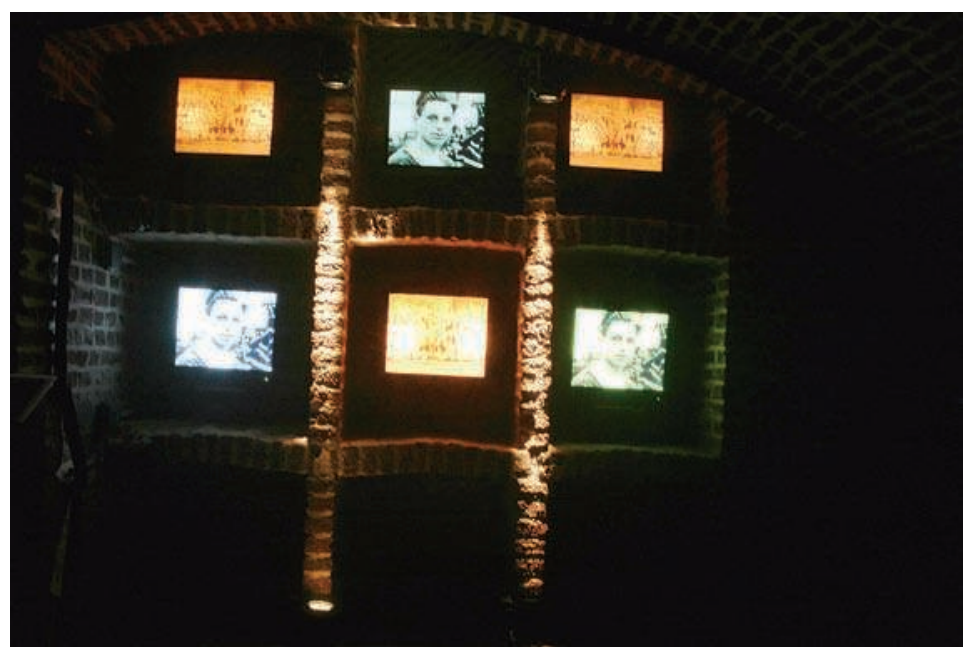

La mémoire ouvrière est sommairement évoquée dans le parcours de la maison de l'Eau : un dispositif multimédia situé dans l'une des caves de la maison de Bonvoisin rappelle

la dureté du travail et les conditions de vie des travailleurs, de façon très poétique. Un peu trop, peut-être. (c) N. Drouguet rétrospective de la mode ou l'importance de l'eau dans l'organisme humain. Scénographiquement parlant, le parcours consacré à l'eau est tout à fait différent du CTLM. Ici, pas de reconstitution mais des évocations multimédias de la Vesdre que l'on peut qualifier d'immersives : le visiteur est tantôt « plongé » dans une ambiance aquatique et écoute la rivière qui prend la parole pour lui raconter son histoire, tantôt il la survole, comme s'il était un oiseau suivant le cours de l'eau. Ces dispositifs spectaculaires paraissent plus sobres qu'au CTLM, cependant, ici, c'est la technologie qui s'affiche. Le parcours multimédia « baigne dans un monde de lumières, de sonorités, d'imageries et de jeux » (5), comme l'affirme le scénographe, Yves Durant, concepteur de l'exposition. Les autres salles ou dispositifs sont plutôt didactiques et ludiques. Ils proposent des jeux multimédias, des bornes interactives à interroger, des maquettes à manipuler... Une salle est réservée aux animations pédagogiques. Enfin, la dernière partie de l'exposition est consacrée à la famille de Bonvoisin, dont les descendants ont légué le bâtiment à la ville. Un arbre généalogique intitulé Six cents ans de dynamisme familial ouvre sur un salon qui "restitue l'ambiance des demeures patriciennes des barons de la laine", où sont présentés des œuvres et des objets de la collection familiale.

\section{Une offre complétée par un parcours en ville}

Ces deux centres d'interprétation sont complétés par un parcours urbain, Je file en ville, promenade pour (re)découvrir les rues, la rivière et le patrimoine bâti de Verviers. L'itinéraire est jalonné de machines industrielles qui ont servi au travail de la laine : fouleuse, laveuse, machine à vapeur, léviathan... Par ailleurs, la «capitale wallonne de l'eau » est, comme il se doit, parsemée de fontaines. Cette offre culturelle et touristique est donc parfaitement cohérente. La maison de l'Eau et le CTLM, installés dans des immeubles historiques, situés dans le quartier le plus industrialisé au XIX ${ }^{e}$ siècle, dans une ville qui tend depuis plusieurs années à transformer son visage en mettant en évidence son riche passé, au cœur d'une région baignée par la Vesdre et marquée par plusieurs barrages, dont celui de la Gileppe, apparaissent comme des points de départ pour découvrir et apprécier la région. En ce sens, les équipements décrits sont tout à fait caractéristiques des fonctions des centres d'interprétation, tels qu'on les envisage au niveau international. 
